\title{
Cómo generar conocimiento a partir de la innovación docente: Estudio de las villae romanas en Asturias
}

\section{How to generate knowledge from teaching innovation: Study of the Roman villae in Asturias}

\author{
Diego Piay Augusto ${ }^{1}$ \\ Fecha de recepción: 09/03/2020; Fecha de revisión: 16/04/2020; Fecha de aceptación: 05/09/2020 \\ Cómo citar este artículo: \\ Piay, D., (2020). Cómo generar conocimiento a partir de la innovación docente. Revista de Innovación y \\ Buenas Prácticas Docentes,9(2), 71-81. \\ Autor de Correspondencia: piaydiego@uniovi.es

\begin{abstract}
Resumen:
El proyecto que se describe nació con el firme propósito de introducir al alumnado de 20 curso del grado de Historia de la Facultad de Filosofía y Letras de la Universidad de Oviedo, que cursa la materia Historia Antigua de Roma, en los resortes de la investigación histórica, acercándolos al estudio de un fenómeno de gran interés para la historia antigua de Asturias como lo es la difusión de las villae (haciendas de carácter rural propiedad de individuos pudientes de la sociedad romana).

Durante el proyecto que se describirá, el alumnado, asistido en todo momento por el docente, aprendió el funcionamiento de los catálogos arqueológicos de Asturias, vaciando la información de interés para el cometido que nos habíamos marcado; elaboró fichas, empleando una base de datos creada ex profeso para este proyecto; revisó y trabajó con la bibliografía de referencia; y expuso los resultados de la investigación de forma coherente. Todo ello persiguiendo una triple motivación: la superación de la asignatura a partir del desarrollo de un proyecto motivador; la experiencia altamente formativa de trabajar con el método histórico; y el fin último del proyecto: la publicación de los resultados junto con el equipo docente, que supervisó toda la actividad.
\end{abstract}

Palabras clave: conocimiento, historia antigua, innovación, publicación

\begin{abstract}
:
The project described was born with the firm purpose of introducing the 2nd year students of the History degree of the Faculty of Philosophy and Letters of the University of Oviedo, who are studying Ancient History of Rome, in the springs of historical research, bringing them closer the study of a phenomenon of great interest to the ancient history of Asturias such as the spread of the villae (rural estates owned by wealthy individuals in Roman society).

During the project to be described, the students, assisted at all times by the teacher, learned the operation of the archaeological catalogs of Asturias, getting the information of interest for the task that we had set ourselves; He produced files, using a database created specifically for this project; reviewed and worked with the reference bibliography; and presented the results of the investigation in a coherent way. All this pursuing a triple motivation: the overcoming of the subject from the development of a motivating project; the highly formative experience of working with the historical method; and the final aim of the project: the publication of the results together with the teaching team, who supervised all the activity.
\end{abstract}

Key Words: knowledge, ancient history, innovation, publication

\footnotetext{
1 Universidad de Oviedo (España), piaydiego@uniovi.es; CÓDIGO ORCID: 0000-0002-0473-1383
} 


\section{INTRODUCCIÓN}

La asignatura Historia Antigua de Roma tiene por objetivo ahondar en los procesos históricos que se desarrollaron en el mundo mediterráneo desde la fundación de Roma (753 aC) hasta las transformaciones que tuvieron lugar durante el inicio de la Antigüedad Tardía, a partir del siglo III dC, partiendo del estudio de los tres períodos en los que tradicionalmente se divide la historia de esta civilización: república, monarquía e imperio. Dentro de la dilata historia de la ciudad eterna ocupan un lugar fundamental las villae, pues representan una consecuencia de la evolución del poblamiento romano en la península y en todo el imperio (Sfameni, 2006), permaneciendo incluso en momentos posteriores con diferentes utilidades (Chavarría, 2007). El interés por el estudio del caso asturiano está, por tanto, justificado, porque permite además definir las singularidades propias de este espacio geográfico y compararlas con las situaciones conocidas en otras regiones de la península ibérica y del imperio. Hay que tener en cuenta que la cuestión de la romanización del noroeste ha hecho correr ríos de tinta, y se han siempre construido esquemas interpretativos basados en una presencia romana minoritaria con respecto a otras regiones de Hispania o, al menos, una romanización diferente. En este sentido, el estudio de las villas romanas puede ayudar a arrojar algo de luz en relación a este proceso cubriendo, además, el vacío existente en relación a este período en lo que a publicaciones científicas se refiere. Se trata, en realidad, de un mal endémico de la arqueología en el noroeste de España, en la cual se favorecen habitualmente las excavaciones de yacimientos de la edad del hierro (castros) por cuestiones ideológicas, primando el estudio de las poblaciones autóctonas que habitaban en Asturias y en Galicia antes de la finalización de las guerras cántabras (19 aC), que supuso la anexión definitiva de Hispania al Imperio romano. Consecuencia de ello es la ausencia de proyectos de investigación relacionados con las villas romanas promocionados por las administraciones públicas, situación que deriva en la práctica total ausencia de publicaciones científicas y, finalmente, en la nula producción de conocimiento científico sobre las mismas.

Este es el contexto arqueológico y social en el que se marca el presente proyecto, que trata de concienciar al alumnado de la problemática que supone desarrollar una investigación sobre la romanización en Asturias, persiguiendo un doble objetivo desde el punto de vista docente: por una parte, poner en práctica nuevas estrategias de aprendizaje, teniendo en cuenta que la alternativa a las clases teóricas magistrales suele ser, normalmente, el uso abusivo de actividades basadas en comentarios de textos, la visualización de documentales o, excepcionalmente, visitas a centros museísticos o yacimientos arqueológicos. Estas iniciativas son insuficientes, y se han revelado como tediosas e incapaces de despertar la motivación en el alumnado a corto o medio plazo. El segundo objetivo que se perseguía con el desarrollo de este proyecto era paliar el escaso bagaje investigador de los estudiantes, que se pone de manifiesto cada vez que se les exige la realización de actividades escritas evaluables a lo largo del curso. El alumnado arrastra año tras año estas carencias, hasta que llega el momento de emprender la realización de trabajos de fin de grado o tesis doctorales. Es entonces cuando a las dificultades asociadas a una investigación en profundidad, se añaden las deficiencias en la metodología empleada, consecuencia directa de no haber recibido ningún tipo de formación orientada a la realización de publicaciones de carácter científico.

Como fin último, se buscaba que el alumnado tomase conciencia de la importancia didáctica de la historia como ciencia fundamental para escrutar el conocimiento del pasado, pues es la única herramienta válida para comprender el presente y afrontar el futuro. En este sentido, era inevitable que para cimentar su sensibilización se partiese de la aplicación rigurosa del método histórico hasta alcanzar la producción científica resultante. 


\section{DESARROLLO DE LA EXPERIENCIA DE INNOVACIÓN}

Teniendo en cuenta la problemática descrita, el diseño del proyecto de innovación docente se configuró para lograr la consecución de una serie de objetivos muy ambiciosos. El proyecto ideado debía mejorar significativamente la docencia impartida en cursos precedentes; motivar al alumnado ofreciéndole una actividad innovadora, centrada en su región de origen y que tendría una cierta repercusión social; y, por último, mejorar el conocimiento de las villas romanas en Asturias con una publicación que ofreciese un catálogo actualizado de este tipo de asentamientos y las conclusiones que se podían derivar de los resultados obtenidos.

\subsection{Diseño}

El proyecto de innovación docente fue realizado por 4 profesores ( 3 de la Universidad de Oviedo y uno de la Universidad de Salamanca), y se articuló en 5 fases que se desarrollaron durante el primer semestre del curso 2019-2020. La sexta fase (publicación de los resultados) está en ejecución, y el compromiso es que el volumen monográfico sobre las villas romanas en Asturias se publique antes de la finalización del año 2020.

En la Figura 1 se enumeran las actividades desarrolladas y se aporta la explicación de cada una de ellas:

\begin{tabular}{|c|c|c|}
\hline FASE & TIPO DE ACTIVIDAD & EXPLICACIÓN \\
\hline $\mathrm{I}$ & $\begin{array}{l}\text { Vaciado de la información de las fichas de } \\
\text { yacimientos arqueológicos } \\
\text { Equipo docente: un profesor. }\end{array}$ & $\begin{array}{l}\text { Se repartieron las fichas } \\
\text { atendiendo a la cercanía de } \\
\text { cada alumno/a con respecto } \\
\text { al yacimiento de estudio }\end{array}$ \\
\hline II & $\begin{array}{l}\text { Compilación de la Base de Datos generada para el } \\
\text { proyecto de innovación } \\
\text { Equipo docente: un profesor. }\end{array}$ & $\begin{array}{l}\text { Se definieron con el } \\
\text { alumnado los campos que } \\
\text { debía incluir en función de los } \\
\text { datos de las fichas }\end{array}$ \\
\hline III & $\begin{array}{l}\text { Estudio de las fuentes clásicas que mencionan las } \\
\text { villas romanas } \\
\text { Equipo docente: un profesor. }\end{array}$ & $\begin{array}{l}\text { Elaboración de un comentario } \\
\text { de texto sobre la evolución } \\
\text { del concepto de villa en los } \\
\text { autores clásicos }\end{array}$ \\
\hline IV & $\begin{array}{l}\text { Recopilación y lectura de la bibliografía existente } \\
\text { sobre las villas en Asturias } \\
\text { Equipo docente: un profesor. }\end{array}$ & $\begin{array}{l}\text { Tarea grupal con elaboración } \\
\text { de un estado de la cuestión } \\
\text { sobre el estudio de las villas } \\
\text { en Asturias }\end{array}$ \\
\hline $\mathrm{V}$ & $\begin{array}{l}\text { Visita guiada a la Villa romana de Veranes a cargo } \\
\text { del equipo docente } \\
\text { Equipo docente: dos profesores. }\end{array}$ & $\begin{array}{l}\text { Explicación de la historia de } \\
\text { las } \\
\text { arqueológicas y excavaciones } \\
\text { yacimiento }\end{array}$ \\
\hline VI & $\begin{array}{l}\text { Publicación de una monografía con los resultados } \\
\text { Equipo docente completo. }\end{array}$ & $\begin{array}{l}\text { Incluye apartados } \\
\text { introductorios, catálogo de } \\
\text { villas, planimetría y capítulos } \\
\text { introductorios }\end{array}$ \\
\hline
\end{tabular}

Figura 1. Fases del proyecto de innovación docente 


\subsection{Objetivos}

Como ya se ha apuntado de forma sucinta, el proyecto se configuró con la intención de cumplir una serie de objetivos muy concretos, acomodados a los destinatarios (Pérez Juste, 269):

- Mejorar la docencia impartida: Tras llevar a cabo una reflexión sobre el programa educativo de la asignatura (Pérez Juste, 2006, p. 266 y ss.), y en particular, sobre los mecanismos empleados para sustituir o complementar las clases teóricas, resulta evidente que estos son cada vez menos apropiados. El proyecto de innovación docente que aquí se presenta se llevó a cabo durante las clases prácticas, como complemento a las clases teóricas. El método buscaba concienciar al alumnado en la idea de que el conocimiento histórico no es algo estático, sino que evoluciona constantemente con la construcción de nuevos paradigmas historiográficos que responden al contexto cultural, político, social e histórico en el que surgen. Es fundamental que comprendan que los contenidos de las clases teóricas están en continuo estudio, y que muchos de los conceptos y teorías que en el pasado se estudiaban como si se tratase de verdades absolutas hoy se discuten (un caso paradigmático es la denominada "crisis del siglo III". Por lo tanto el modelo educativo que se trataba imponer se basaba en el desarrollo de la actitud crítica entre los estudiantes.

- Lograr la motivación del alumnado: Este principio es fundamental, pues un alumno motivado implica un profesor motivado y a la inversa. Involucrar al alumnado no implica captar su atención por la presencia de una evaluación final que debe superar, y que en general considera arbitraria y azarosa (Cano, 2015). Involucrar al alumnado significa lograr su implicación en un proyecto más allá de la superación de la asignatura. Elegir un tema basado en su propia historia (la de Asturias) y, cuando fue posible, de su propia parroquia o núcleo poblacional, se reveló como una estrategia de lo más adecuada. Aun cuando el nivel de implicación varía dependiendo del alumno, la gran mayoría realizó actividades complementarias por voluntad propia (visitas a museos de la zona, entrevistas con personas ancianas que recordaban el hallazgo de determinados materiales durante labores agrícolas, etc.)

- Publicación de una monografía. Directamente relacionado con el apartado anterior. Un proyecto que tiene un principio, un desarrollo y un final que supone una cierta repercusión social y un mérito curricular complementa la natural alegría producida por la superación de la asignatura. No puede olvidarse, además, la satisfacción inherente a una investigación concluida, en la que el alumno percibe, realmente, que ha aportado su esfuerzo a un bien común, que posibilitará, en el futuro, un conocimiento más preciso de una parte de nuestro pasado poco conocida.

\subsection{Competencias desarrolladas}

El proyecto de innovación docente se basaba en tres principios fundamentales:

- En primer lugar, se buscaba que el alumnado conociese de primera mano el modo en que se lleva a cabo una investigación de carácter arqueológico, partiendo de la revisión de los catálogos de yacimientos existentes, de los cuales es necesario extraer la información de utilidad y emplear correctamente los datos obtenidos. En este sentido, puede afirmarse que el alumnado comprendió el funcionamiento de los diferentes catálogos consultados, y sabrá emprender de forma autónoma estudios de este tipo con perfectas garantías. Durante el desarrollo de la Fase I se realizó un vaciado masivo de la información de interés y se llevó a cabo un debate general se discutió el modo en que debían ser vaciados los datos para su mayor aprovechamiento.

- En segundo lugar, se trataba de diseñar un recorrido metodológico coherente y sistemático, que permitiese al alumnado conocer los pasos que debe seguir una 
investigación desde sus inicios hasta su final, con la publicación de los resultados d ela misma. Diseñar un recorrido metodológico que permita al alumnado conocer los pasos que debe seguir una investigación desde sus inicios hasta su final, con la publicación de los resultados de la misma. Desde este punto de vista puede decirse que el alumnado no dejó de manifestar su satisfacción durante el desarrollo de toda la actividad, pues era la primera vez que se enfrentaban a una investigación histórica como tal, superando y desarrollando todas las etapas del proceso delineado.

- Por último, algunas de las actividades -en concreto, las desarrolladas durante las fases IV y V- se realizaron en grupo, a fin de fomentar la labor de equipo y la competitividad sana entre el alumnado. Los estudiantes competían entre sí y valoraban de forma crítica el trabajo de sus compañeros. Dentro de cada grupo, sus integrantes trabajaron de forma serie y responsable para realizar las tareas señaladas, y posteriormente las expusieron oralmente en el aula ante el resto del alumnado.

\subsection{Recursos empleados}

Para el desarrollo del proyecto se emplearon diversas herramientas que facilitaron su puesta en práctica. Los equipos informáticos y los dispositivos móviles fueron necesarios durante toda la actividad y sirvieron para llevar a cabo el vaciado de las fichas del catálogo de yacimientos arqueológicos asturianos, para la elaboración de contenidos, y para la búsqueda en hemerotecas digitales y motores de búsqueda de internet de cualquier información relacionada con cada una de las villas que configuran el catálogo. En este sentido, la familiaridad del alumnado con este tipo de dispositivos posibilitó la localización de informaciones de difícil acceso con búsquedas simples en motores como google.

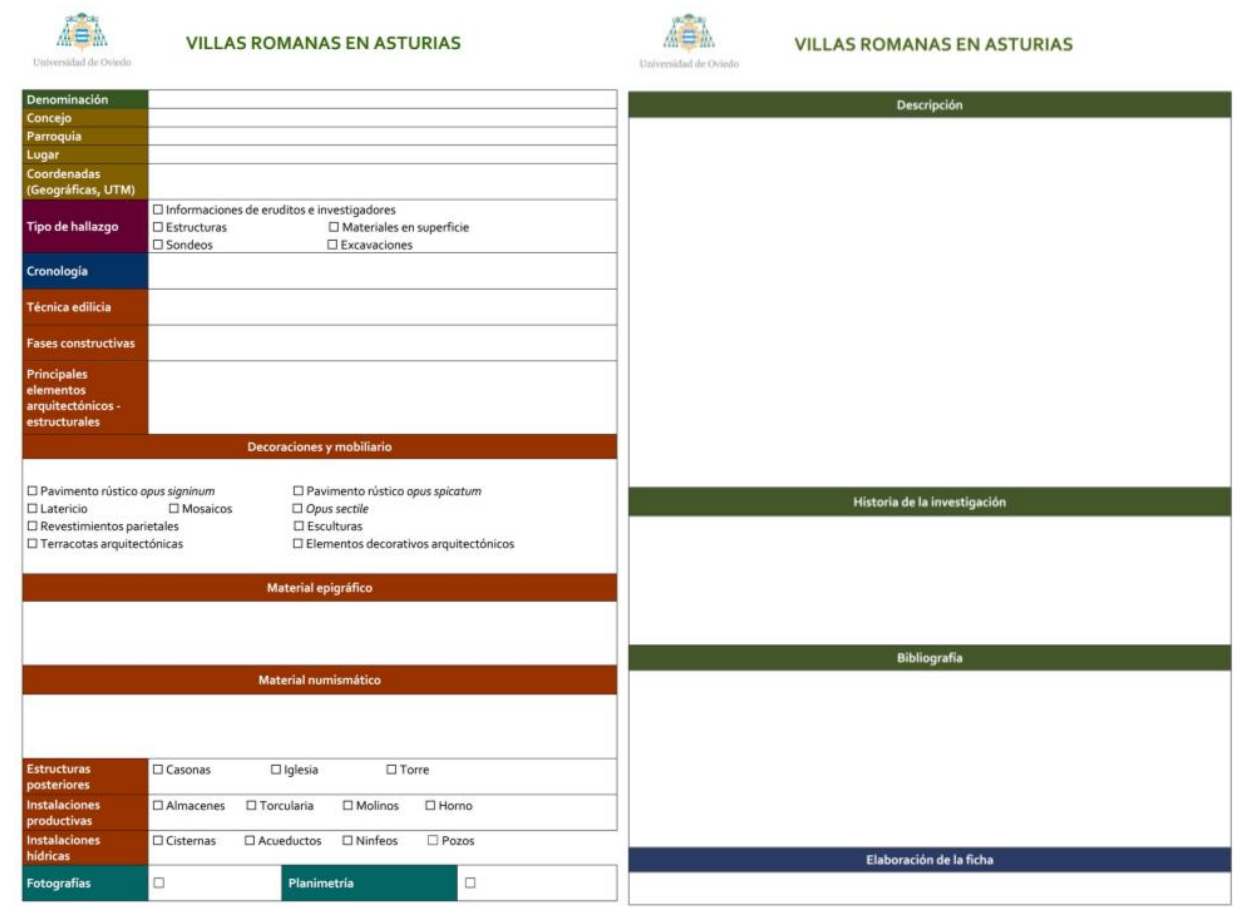

Figura 2. Modelo de ficha para catalogación de las villae asturianas elaborado durante la realización del proyecto de innovación docente 
Además, se usaron programas para la realización de bases de datos, y también software específico para la elaboración de la planimetría general y específica de cada villa. En este sentido, se colaboró con el Departamento de Geografía de la Universidad de Oviedo, fomentando la interdisciplinariedad, tan valiosa en los estudios de carácter arqueológico e histórico.
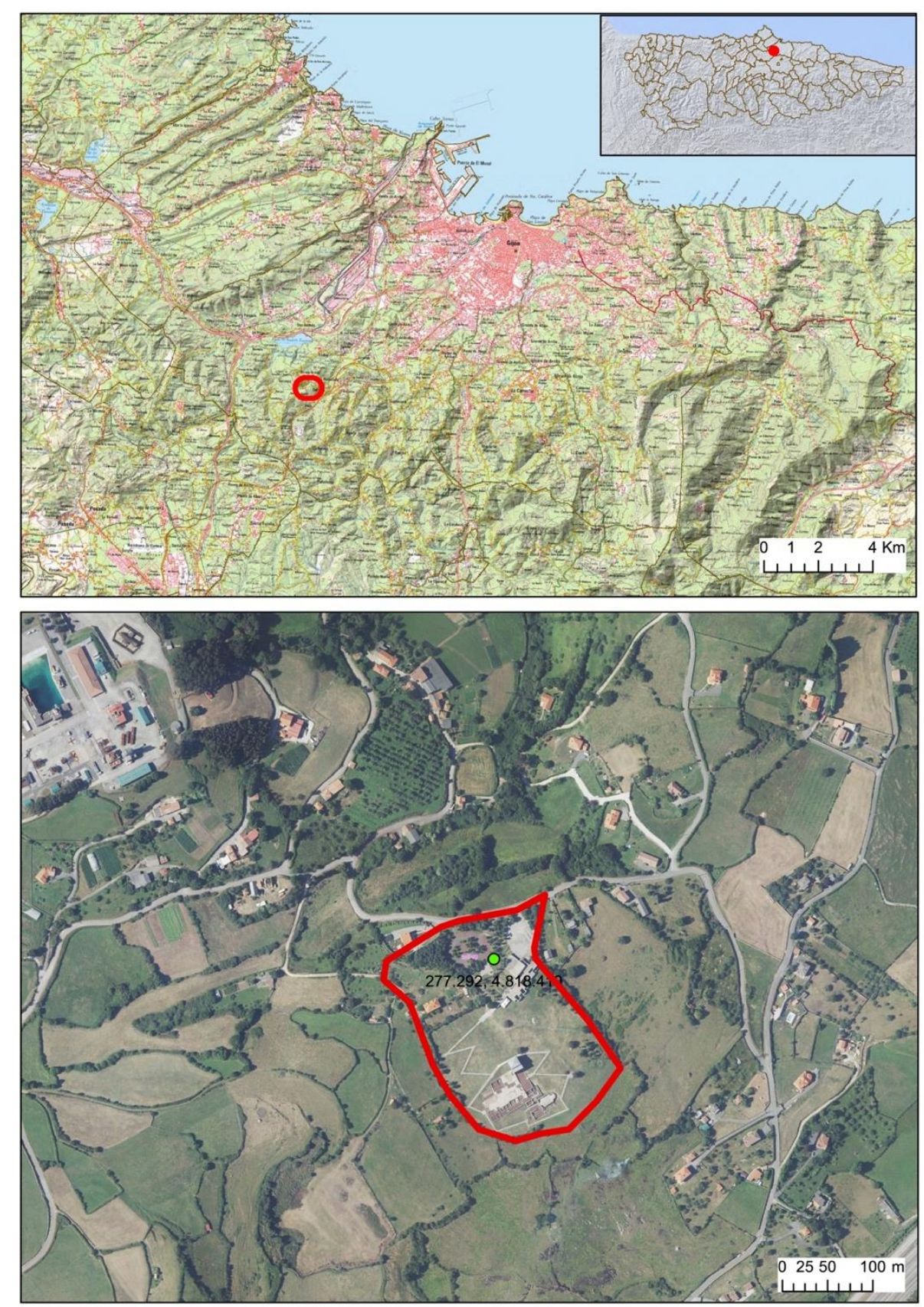

Figura 3. Modelo de hoja planimétrica de la villa de Veranes creada durante el desarrollo del proyecto 
Para la evaluación de las diferentes actividades se diseñó una matriz de valoración o rúbrica, con dos ejes en los que se especificaban los criterios de ejecución de las tareas y la escala de evaluación (Cano, 2015). Para obtener una visión global del grado de satisfacción de la actividad se realizó un cuestionario a todos los participantes en el proyecto, incluyendo al profesorado, en el cual se valoraba entre 0 y 5 el grado de satisfacción empleando preguntas relacionadas con el proyecto y su ejecución.

Tabla 1.

Cuestionario creado ex novo para la autoevaluación del equipo docente.

\begin{tabular}{|c|c|c|c|c|c|}
\hline CRITERIOS & $9-10$ & $7-8$ & $5-6$ & 3-4 & $1-2$ \\
\hline $\begin{array}{c}\text { Ser capaz de } \\
\text { aplicar el método } \\
\text { científico aplicado } \\
\text { al estudio de la } \\
\text { Historia y } \\
\text { Arqueología }\end{array}$ & $\begin{array}{l}\text { Analiza y } \\
\text { procesa } \\
\text { correctamente } \\
\text { la información } \\
\text { proveniente de } \\
\text { diversas } \\
\text { fuentes }\end{array}$ & $\begin{array}{c}\text { Con } \\
\text { frecuencia } \\
\text { analiza y } \\
\text { procesa } \\
\text { correctamente } \\
\text { la información } \\
\text { proveniente de } \\
\text { diversas } \\
\text { fuentes }\end{array}$ & $\begin{array}{l}\text { A veces analiza } \\
\text { y procesa de } \\
\text { manera correcta } \\
\text { la información } \\
\text { proveniente de } \\
\text { diversas fuentes } \\
\text { aunque sin } \\
\text { profundizar } \\
\text { demasiado }\end{array}$ & $\begin{array}{c}\text { No suele } \\
\text { analizar ni } \\
\text { procesar la } \\
\text { información } \\
\text { proveniente } \\
\text { de diversas } \\
\text { fuentes ni } \\
\text { profundizar } \\
\text { en ellas }\end{array}$ & $\begin{array}{c}\text { Nunca } \\
\text { analiza ni } \\
\text { procesa la } \\
\text { información } \\
\text { proveniente } \\
\text { de diversas } \\
\text { fuentes ni } \\
\text { profundizar } \\
\text { en ellas }\end{array}$ \\
\hline $\begin{array}{c}\text { Comprende y sabe } \\
\text { aplicar la } \\
\text { metodología } \\
\text { divulgativa } \\
\text { científica aplicable } \\
\text { en publicaciones } \\
\text { de impacto }\end{array}$ & $\begin{array}{c}\text { Comprende y } \\
\text { elabora } \\
\text { producción } \\
\text { científica de } \\
\text { gran calidad }\end{array}$ & $\begin{array}{c}\text { Con } \\
\text { frecuencia } \\
\text { comprende y } \\
\text { elabora } \\
\text { producción } \\
\text { científica de } \\
\text { gran calidad }\end{array}$ & $\begin{array}{c}\text { Parece } \\
\text { comprender } \\
\text { aunque con } \\
\text { gran esfuerzo } \\
\text { le cuesta } \\
\text { elaborar } \\
\text { producción } \\
\text { científica de } \\
\text { gran calidad }\end{array}$ & $\begin{array}{c}\text { No parece } \\
\text { comprender } \\
\text { la } \\
\text { metodología } \\
\text { científica } \\
\text { divulgativa ni } \\
\text { es capaz de } \\
\text { desarrollar } \\
\text { textos } \\
\text { científicos } \\
\end{array}$ & $\begin{array}{c}\text { No } \\
\text { comprende la } \\
\text { metodología } \\
\text { científica } \\
\text { divulgativa ni } \\
\text { es capaz de } \\
\text { desarrollar } \\
\text { textos } \\
\text { científicos }\end{array}$ \\
\hline $\begin{array}{c}\text { Capacidad de } \\
\text { trabajar en equipo } \\
\text { y desarrollar } \\
\text { competencias } \\
\text { como "aprender a } \\
\text { aprender", } \\
\text { "iniciativa y espíritu } \\
\text { emprendedor", } \\
\text { "competencia } \\
\text { digital”, "conciencia } \\
\text { y expresión } \\
\text { cultural” y } \\
\text { "comunicación } \\
\text { lingüística" }\end{array}$ & $\begin{array}{c}\text { Desarrolla } \\
\text { todas las } \\
\text { competencias } \\
\text { de aprendizaje } \\
\text { propuestas y } \\
\text { sabe aportar } \\
\text { tanto al trabajo } \\
\text { en equipo } \\
\text { como a su } \\
\text { aprendizaje } \\
\text { individual }\end{array}$ & $\begin{array}{c}\text { Intenta } \\
\text { desarrollar } \\
\text { todas las } \\
\text { competencias } \\
\text { de aprendizaje } \\
\text { propuestas y } \\
\text { sabe aportar } \\
\text { tanto al trabajo } \\
\text { en equipo } \\
\text { como a su } \\
\text { aprendizaje } \\
\text { individual } \\
\text { aunque con } \\
\text { alguna } \\
\text { dificultad }\end{array}$ & $\begin{array}{c}\text { No es capaz de } \\
\text { desarrolla todas } \\
\text { las } \\
\text { competencias } \\
\text { de aprendizaje } \\
\text { propuestas y le } \\
\text { cuesta el } \\
\text { trabajo en } \\
\text { equipo así } \\
\text { como el } \\
\text { individual }\end{array}$ & $\begin{array}{c}\text { No es capaz } \\
\text { de desarrolla } \\
\text { casi ninguna } \\
\text { de las } \\
\text { competencia } \\
\text { s de } \\
\text { aprendizaje } \\
\text { propuestas y } \\
\text { no aporta al } \\
\text { trabajo en } \\
\text { equipo ni al } \\
\text { suyo } \\
\text { individual }\end{array}$ & $\begin{array}{c}\text { No es capaz } \\
\text { de } \\
\text { desarrolla } \\
\text { ninguna } \\
\text { competenci } \\
\text { as de } \\
\text { aprendizaje } \\
\text { propuestas } \\
\text { ni sabe } \\
\text { trabajar de } \\
\text { manera } \\
\text { individual y } \\
\text { en equipo }\end{array}$ \\
\hline $\begin{array}{l}\text { Aplicar estrategias } \\
\text { dinámicas con } \\
\text { metodologías } \\
\text { innovadoras que } \\
\text { hagan aumentar la } \\
\text { participación y } \\
\text { asistencia al aula }\end{array}$ & $\begin{array}{c}\text { El alumno } \\
\text { asiste a todas } \\
\text { las actividades } \\
\text { propuestas y } \\
\text { participa } \\
\text { activamente } \\
\text { demostrando } \\
\text { gran interés }\end{array}$ & $\begin{array}{c}\text { El alumno } \\
\text { asiste a casi } \\
\text { todas todas } \\
\text { las actividades } \\
\text { propuestas, } \\
\text { participa a } \\
\text { menudo y } \\
\text { tiene interés }\end{array}$ & $\begin{array}{c}\text { El alumno } \\
\text { asiste } \\
\text { ocasionalmente } \\
\text { al aula y sus } \\
\text { participaciones } \\
\text { son puntuales. } \\
\text { El interés es } \\
\text { ocasional } \\
\end{array}$ & $\begin{array}{l}\text { El alumno } \\
\text { casi nunca } \\
\text { asiste al aula } \\
\text { y apenas } \\
\text { participa y } \\
\text { demuestra } \\
\text { interés }\end{array}$ & $\begin{array}{c}\text { El alumno no } \\
\text { ha asistido a } \\
\text { ninguna de las } \\
\text { actividades } \\
\text { propuestas y } \\
\text { por tanto su } \\
\text { participación e } \\
\text { interés es nulo }\end{array}$ \\
\hline
\end{tabular}


Tabla 2.

Cuestionario creado ex novo para la autoevaluación del equipo docente.

\begin{tabular}{|c|c|c|}
\hline \multicolumn{3}{|c|}{ AUTOEVALUACIÓN DEL EQUIPO DOCENTE (4 respuestas) } \\
\hline Preguntas planteadas & Evaluación & $\%$ \\
\hline $\begin{array}{c}\text { ¿Se han obtenido los objetivos } \\
\text { propuestos? }\end{array}$ & 012345 & $\begin{array}{ll}50 \% & 5 \\
50 \% & 4 \\
\end{array}$ \\
\hline ¿Se ha innovado realmente en el aula? & 012345 & $\begin{array}{ll}75 \% & 5 \\
25 \% & 4 \\
\end{array}$ \\
\hline $\begin{array}{c}\text { ¿Se ha logrado una participación activa } \\
\text { del alumnado? }\end{array}$ & 012345 & $100 \%$ \\
\hline $\begin{array}{c}\text { ¿Se han comprendido los contenidos } \\
\text { propuestos? }\end{array}$ & 012345 & $\begin{array}{l}75 \% \\
25 \%\end{array}$ \\
\hline $\begin{array}{l}\text { Nivel de satisfacción general con el } \\
\text { resultado del PID }\end{array}$ & 012345 & $100 \%$ \\
\hline
\end{tabular}

Tabla 3.

Cuestionario creado ex novo para la evaluación por parte del alumnado.

\begin{tabular}{cccc}
\hline \multicolumn{3}{c}{ EVALUACIÓN POR PARTE DEL ALUMNADO (74 respuestas) } & \\
\hline Preguntas planteadas & Evaluación & $\%$ \\
\hline $\begin{array}{c}\text { ¿Habrías preferido desarrollar un tipo de } \\
\text { docencia más "tradicional"? }\end{array}$ & 012345 & $95 \%$ & 0 \\
¿Crees que el profesor ha conseguido & 012345 & 1 \\
motivar al alumnado? & & $25 \%$ & 4 \\
\hline ¿Consideras el proyecto útil para la & & $75 \%$ & 5 \\
\hline asignatura? & & 512345 & 5 \\
& & $25 \%$ & 2 \\
& & $25 \%$ & 4 \\
\hline
\end{tabular}




\section{RESULTADOS}

La actividad se emprendió con cierto temor por su carácter novedoso y el volumen de estudiantes matriculados (84). Se buscaba generar en los estudiantes la pasión por la historia y su conocimiento, dado que es natural observar cómo el alumnado se debate entre un futuro profesional poco halagüeño y un irracional deseo por conocer el pasado. Se ha perseguido que el segundo factor de este dilema primase sobre el primero. $Y$ aunque, como es natural, no todos los estudiantes alcanzaron el mismo nivel de implicación, una parte del mismo ha querido seguir indagando sobre el tema de estudio de este proyecto, constituyendo la mejor evidencia de su éxito. Y además, se ha generado conocimiento científico, pues se han ampliado y actualizado los datos existentes sobre las villas romanas en Asturias, no analizados globalmente desde la última monografía que aborda esta temática (Fernández Ochoa, 1982) y un artículo posterior (Santos, 1984). Especial valor se ha revelado en el desarrollo de una actividad de estas características con la potencialidad de contar con 84 puntos de vista diferentes, con comentarios de todo tipo algunos de ellos dotados de gran perspicacia, pero, en cualquier caso, todos ellos útiles y que evidencian cómo los mismos temas analizados por personas diferentes dan a lugar a conclusiones variopintas que pueden en ocasiones complementarse.

Se ha comprometido ya la publicación para este año de la monografía con una casa editorial especializada en arqueología. La aparición de este volumen supondrá un avance significativo en el conocimiento de las villas romanas en Asturias, investigación que tan solo se ha visto enriquecida en los últimos años con publicaciones relacionadas con la villa de Veranes (Fernández Ocho et alii, 1982, 1992, 2004; 2005; 2008; 2013; García, 2008), la más indagada de todo el territorio asturiano.

Para la realización del volumen monográfico en preparación se han revisado todos los yacimientos catalogados en el territorio asturiano; se han definido en una base de datos los criterios específicos para su inclusión en el elenco de villas; se ha realizado una exhaustiva búsqueda bibliográfica en las bibliotecas y hemerotecas para completar los datos disponibles, aportando informaciones actualizadas sobre cada uno de los yacimientos; se ha elaborado una planimetría general y de detalle; se han revisado las fuentes clásicas que definen el asentamiento romano conocido como "villa", permitiéndonos conocer la evolución del término y la multitud de realidades a las que puede hacer referencia (desde una simple factoría sin ornamentos, hasta una rica hacienda con hipocaustos y estancias decoradas con mosaicos y pinturas parietales); y, por último, se ha revisado toda la bibliografía existente sobre las villas romanas en Asturias, elaborando un estado de la cuestión de la investigación sobre este tema de estudio, que como ya se ha dicho, adolece de obras de conjunto desde hace más de 30 años.

El trabajo desarrollado contribuirá, sin duda, a aportar un hito significativo en el curriculum vitae de los estudiantes, que han desarrollado todas las etapas del método histórico (selección de un tema de estudio, recopilación de toda la documentación disponible, lectura de las fuentes clásicas, recopilación bibliográfica y publicación de los resultados).

Gracias al desarrollo de esta actividad el alumnado estará preparado para abordar en el futuro próximo la realización de sus trabajos de fin de grado y sus tesis doctorales, reforzados con la formación adquirida durante una experiencia práctica en la que han tenido una participación activa, y en la que han seguido un proceso de investigación desde el principio y hasta el final. 


\section{CONCLUSIONES}

El proyecto expuesto es totalmente innovador en materia docente y, en el ámbito histórico, su carácter pionero es aún más destacable. Se combate, en primer lugar, con la sombra del empleo del trabajo del alumnado para los propios fines del docente, que pronto se ha visto despejada por el diálogo constante, el hecho de compartir con el alumnado fichas diseñadas para este proyecto, el índice del volumen, o los planos generados de los diferentes yacimientos arqueológicos. El punto fuerte de este proyecto es haber compartido una actividad innovadora con alumnos y alumnas motivados por estudiar restos arqueológicos que forman parte de la historia de su región, elaborando una investigación común, aunando puntos de vista y formaciones académicas a menudo diversas. El punto débil ha sido, sin duda, la falta de financiación para realizar una iniciativa que debería ser la conclusión natural de este proyecto: emprender la excavación de una villa romana en la comunidad de Asturias con el alumnado, para que todos los contenidos teórico-prácticos aprendidos y discutidos, fuesen trasladados a la realidad arqueológica. No obstante, se ha generado un grupo muy implicado y seguirán buscándose alternativas para poder completar en el futuro la actividad desarrollada, tratando de establecer vías de colaboración entre la universidad y entes públicos y privados que puedan satisfacer las exigencias de un alumnado en proceso de aprendizaje y necesitado de proyectos que canalicen sus aspiraciones formativas y que les preparen para su futuro profesional.

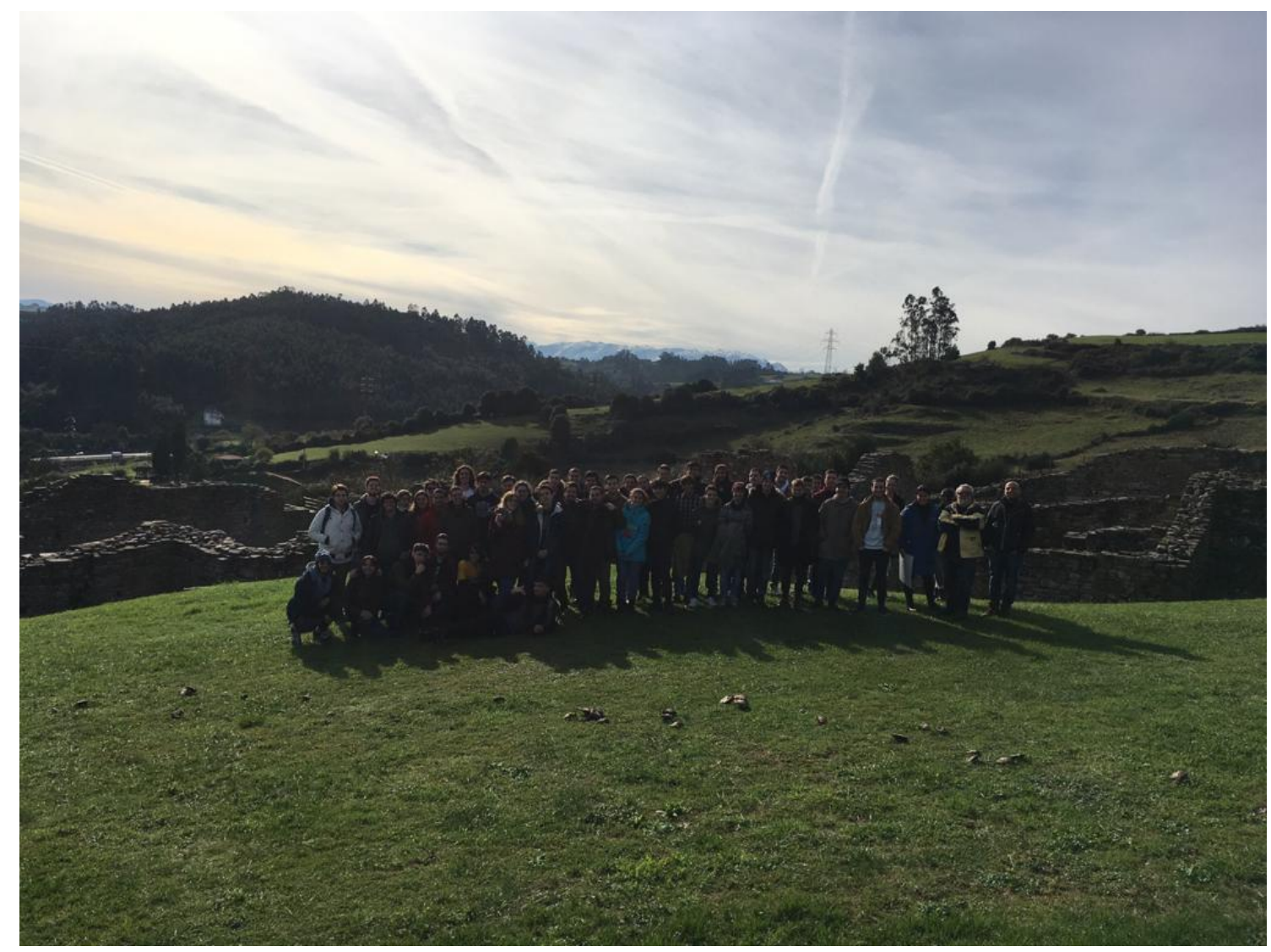

Figura 4. Visita a la villa romana de Veranes realizada el 19 de noviembre de 2019. 


\section{REFERENCIAS}

Blázquez Martínez, J.M. y Blanco Freijeiro, A. (1990). La sociedad del Bajo Imperio en la obra de Salviano de Marsella. Madrid: Real Academia de la Historia.

Cano, E. (2015). Las rúbricas como instrumento de evaluación de competencias en educación superior. ¿Uso o abuso? Profesorado, 19(2), 265-280.

Chavarría, A. y Arce, J. (2006). Villas tardoantiguas en el Mediterráneo occidental. Editorial CSIC- CSIC Press.

Chavarría Arnau, A. (2007). El final de las "villae" en "Hispania" (siglos IV-VII D.C.). Turnhout: Brepols.

Fernández Ochoa, C. F. (1982). Asturias en la época romana. Madrid: Departamento de Prehistoria y Arqueología, Universidad Autónoma de Madrid.

Fernández Ochoa, C. (1992). Asturias en época romana. Madrid: Departamento de Prehistoria y Arqueología. Universidad Autónoma de Madrid.

Fernández Ochoa, C., Gil Sendino, F., y Saco del Valle A. (2004). La villa romana de Veranes. El Complejo rural tardorrromano y propuesta de estudio del territorio. Archivo Español de Arqueología, 77, 197-220.

Fernández Ochoa, C., Gil Sendino, F., Villar Calvo, A., Rodríguez, N., Álvarez Toledo, G. y Morán Fernández, O. (2005). La villa romana de Veranes (Gijón, Asturias). Aportaciones preliminares sobre la transformación funcional del asentamiento en la tardía antigüedad.

Fernández Ochoa, C. F., Entero, V. G., y Sendino, F. G. (Eds.). (2008). Las" villae" tardorromanas en el occidente del Imperio: arquitectura y función. Trea.

Fernández Ochoa, C., Gil Sendino, F., y Salido Domínguez, J. (2013). Nuevas evidencias del cristianismo en Asturias: los crismones de la villa romana de Veranes (Gijón).

García, M. G. (2008). "Intervención en la Villa Astur romana de Veranes en Gijón". Liño: Revista anual de historia del arte, 14, pp. 211-216.

Medina Rivilla, A. y otros (2011). Innovación de la educación y de la docencia. Madrid: Editorial Universitaria Ramón Areces / UNED.

Pérez Juste, R. (2006). Evaluación de programas educativos. Madrid: La Muralla.

Santos Yanguas, N. (1984). Las villas romanas en Asturias. Memorias de historia antigua, 6, 155-174.

Sfameni, C. (2006). Ville residenziali nell'Italia tardoantica. Bari: Edipuglia.

\section{Fuentes clásicas empleadas en el marco del proyecto:}

Cicerón. (2008) Epistolarum ad familiares (J. A. Beltrán, Trad.). Barcelona: Gredos.

Columela. (2004). De Re Rustica (J. I. García Armendariz, Trad.). Barcelona: Gredos. Marcial. (1997). Epigramas (Antonio Ramírez de Verger). Barcelona: Gredos.

Plinio (2017). Cartas (Julián González Fernández). Barcelona: Gredos.

Símaco, (2003). Correspondance. París: Les Belles Lettres.

Varrón. (2010). De Re Rustica (J. I. Cubero Salmerón, Trad.). Sevilla: Consejería de Agricultura y Pesca, Servicio de Publicaciones y Divulgación. 